Radiologe 2012 · 52:977-978

DOI 10.1007/s00117-012-2405-1

Online publiziert: 24. Oktober 2012

(c) Springer-Verlag Berlin Heidelberg 2012

\section{Redaktion: S. Delorme}

\section{Originalpublikation}

Krings T, Mandell DM, Kiehl TR (2011) Intracranial aneurysms: from vessel wall pathology to therapeutic approach. Nat Rev Neurol 7:547-559

Die von Krings et al. 2011 in Nature Reviews Neurology publizierte Übersichtsarbeit beschäftigt sich mit der Pathophysiologie und daraus abgeleitet der Behandlung intrakranieller Aneurysmen.

Die Aneurysmablutung ist mit nur etwa $5 \%$ eine relativ seltene Ursache des Schlaganfalls, aber die häufigste Ursache der atraumatischen Subarachnoidalblutung (SAB) und gehört zu den wichtigsten akuten Notfällen in den Neurofächern. Mit etwa 3\% ist die Prävalenz in-

\section{F.J. Ahlhelm}

Abteilung für Diagnostische und Interventionelle Neuroradiologie,

Klinik für Radiologie und Nuklearmedizin, Universitätsspital Basel

\title{
Intrakranielle Aneurysmen - ein heterogenes Krankheitsbild
}

trakranieller Aneurysmen in der Bevölkerung (bei einem Durchschnittsalter von 50 Jahren) auch nicht gering. Aufgrund der hohen Mortalität und Morbidität müssen bei Patienten, die mit einer Aneurysmablutung symptomatisch werden, eine zügige Diagnostik und zeitnahe Therapie erfolgen, um die gefürchtete und oftmals letal verlau

fende Nachblutung zu verhindern.

Wie die Übersicht anschaulich zeigt, handelt es sich bei intrakraniellen Aneurysmen nicht um ein eigenständiges Krankheitsbild, sondern eine Manifestation verschiedener Erkrankungen der Gefäßwand, was auch unterschiedliche therapeutische Ansätze bedingt. Dabei ist zu betonten, dass auch wenn bis heute im klinischen Alltag die Gefäßaussackung häufig im Vordergrund steht, die eigentliche Pathologie in der Gefäßwand liegt. Die Übersicht unterscheidet hier 8 verschiedene Typen (klassisch sakkuläre Aneurysmen, segmentale Ektasien, Dissektionsaneurysmen, intramurale Blutungen, mykotische oder mikrobielle Aneurysmen, immundefizienzassoziierte Aneurysmen, neoplastische und traumatische Aneurysmen).

Am häufigsten sind die klassischen oder auch atherosklerotischen Aneurysmen an den Teilungsstellen der Hirnbasisarterien. Diese Aneurysmen werden auch als klassisch sakkuläre, Bifurkations- oder „Berry“-Aneurysmen bezeichnet. Diese Aneurysmen sind mit Abstand am häufigsten, wobei die Pathogenese noch nicht vollständig geklärt ist. Die frühere Vor-
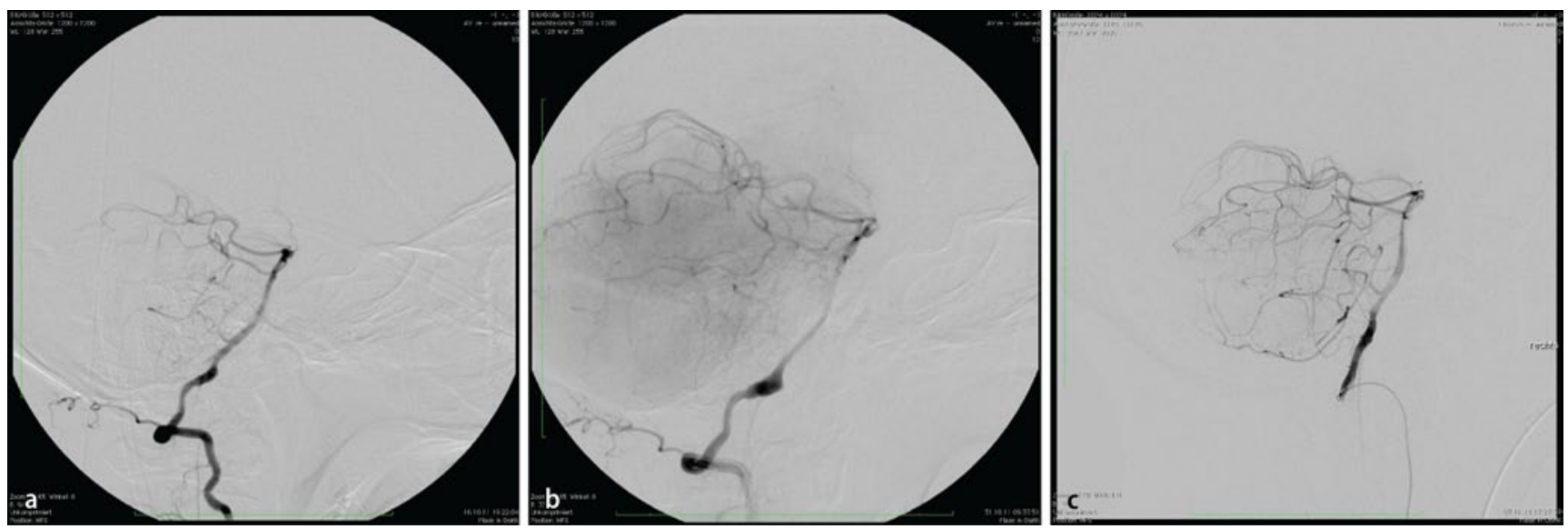

Abb. $1 \Delta$ Stent-alone-Behandlung eines Dissektionsaneurysmas im V4-Segment der rechten Vertebralarterie (VA) bei einem 53-jährigen Patienten mit Fischer-IV-Subarachnoidalblutung. Selektive Darstellung der VA links im seitlichen Strahlengang. Nachweis eines fusiformen V4-Aneurysmas vor (a) und Befundprogredienz in der Verlaufsuntersuchung 2 Wochen später (b). Eine Woche nach Stenteinlage (c) ist die fusiforme Erweiterung in der Mikrokatheteruntersuchung deutlich rückläufig (Stentin-Stent-Behandlung mit insgesamt 3 "closed cell stents", 2-mal Enterprise, einmal Pipeline) 
stellung einer kongenitalen Erkrankung ist heute überholt, wobei zunehmend luminale (hämodynamischer Stress) und abluminale (Veränderungen der Gefäßwand) Faktoren in den wissenschaftlichen Fokus geraten. Diese klassischen Berry-like-Aneurysmen können heute endovaskulär mittels Coiling ( \pm Stenteinlage) oder chirurgisch mittels Clipping in den meisten Fällen erfolgreich behandelt werden. Ein iatrogener Verschluss des aneurysmatragenden Gefäßes ist im Gegensatz $\mathrm{zu}$ früher heute in der Regel nicht mehr notwendig.

Im Gegensatz dazu ist die reine Coilembolisation bei mykotischen, neoplastischen oder traumatischen Aneurysmen und Dissektionsaneurysmen kontraindiziert. Neben dem chirurgischen oder endovaskulären Gefäßverschluss ist hier in ausgewählten Fällen auch manchmal ein konservatives Vorgehen angezeigt. Bei der Behandlung von Dissektionsaneurysmen werden heute auch zunehmend spezielle „Neurostents“ erfolgreich eingesetzt (- Abb. 1).

In Anlehnung an die von Mizutani et al. bereits 1999 in Neurosurgery publizierte Klassifikation von Dissektionsaneurysmen schließt das Review auch seltene Aneurysmaformen wie die mit einer Immundefizienz assoziierte Aneurysmen ein und gibt eine schöne Übersicht über die komplexe Thematik. Ob die segmentale Ektasie ein eigenständiger Typus von Aneurysmen ist oder zu der Gruppe der Dissektionsaneurysmen zählt, ist sicherlich diskutabel. In jedem Fall ist die Übersichtsarbeit allen Kollegen, die sich mit intrakraniellen Aneurysmen beschäftigen, wärmstens ans Herz zu legen.

\section{Korrespondenzadresse}

PD Dr. F.J. Ahlhelm

Abteilung für Diagnostische und Interventionelle Neuroradiologie, Klinik für Radiologie und Nuklearmedizin, Universitätsspital Basel, Petersgraben 4, CH-4031 Basel, Schweiz

frank.ahlhelm@gmail.com

Interessenkonflikt. Der korrespondierende Autor gibt an, dass kein Interessenkonflikt besteht.

\section{Berliner Röntgen-Gesellschaft verleiht Gustav-Bucky-Preis}

Zum ersten Mal hat die Berliner Röntgen-Gesellschaft - Röntgenvereinigung zu Berlin und Brandenburg e.V. den Gustav-Bucky-Preis verliehen. Preisträger sind Dr.-Ing.

Alexander Gussew vom Klinikum der Friedrich-Schiller-Universität Jena und Florian F. Schmitzberger von der Charité Berlin. Dr.-Ing. Alexander Gussew wurde im russischen Perm geboren und schloss 2006 sein Studium zum Diplomingenieur für Elektrotechnik und Informationstechnik an der TU IImenau ab. In seiner Dissertation am Klinikum der Friedrich-Schiller-Universität Jena beschäftigt er sich mit der Darstellung von biochemischen Prozessen während der Schmerzverarbeitung im Gehirn mittels Protonenmagnetresonanzspektroskopie. Seine Erkenntnisse ermöglichen eine Verbesserung der bildgebenden Diagnostik bei chronischen Schmerzen.

Der in Innsbruck, Österreich, geborene Florian Schmitzberger machte 2009 einen MasterAbschluss in Biomedical Informatics an der amerikanischen Stanford University und hat 2010 ein Promotionsstudium an der Berliner Charité aufgenommen. Florian Schmitzbergers Forschungsergebnisse leisten einen wichtigen Beitrag zu einer besseren bildlichen Diagnostik von Brustkrebstumoren und zur Senkung der Strahlenexposition. Mit dem jährlich verliehenen Gustav-BuckyPreis soll der wissenschaftliche Nachwuchs in der Radiologie gefördert werden. Die Bewerbungsfrist für den Gustav-Bucky-Preis 2013 endet am 31.12.2012. Der Namensgeber des Preises, Gustav Bucky wurde am 3. September 1880 in Leipzig geboren. Bekannt wurde er vor allem durch die Erfindung der nach ihm benannten Bucky Blende, einem wichtigen Beitrag zur Reduktion der Streustrahlung und Qualitätsverbesserung in der Röntgendiagnostik. 1933 emigrierte Bucky von Berlin nach New York. Dort war er unter anderem Hochschullehrer am New York College of Medicine sowie am Albert Einstein College of Medicine. Die Röntgenvereinigung zu Berlin und Brandenburg ehrt mit diesem Preis Gustav Bucky sowie seine radiologischen Kollegen, die zwischen 1933 und 1945 aus ihrer Tätigkeit verbannt oder zur Emigration gezwungen wurden.

Quelle: Deutsche Röntgengesellschaft e.V.
1. Internationaler Tag der Radiologie am 8. 11. 2012 Deutsche Röntgengesellschaft (DRG) und die Deutsche Gesellschaft für Radioonkologie (DEGRO) informieren

Moderne Medizin ist ohne Bildgebung nicht mehr vorstellbar. Auch in der Tumorbehandlung ist sie unverzichtbar. Wie groß der Beitrag der Radiologen und Strahlentherapeuten zu Diagnose und Therapie von Krebserkrankungen ist, ist der Öffentlichkeit meist nicht bekannt.

Um mehr Wissen über die Bedeutung der Radiologie und Strahlentherapie zu vermitteln, haben internationale radiologische Fachgesellschaften den Aktionstag ins Leben gerufen. Der 8. November ist ein geschichtsträchtiges Datum: An diesem Tag entdeckte Wilhelm Conrad Röntgen 1895 eine „neue Art von Strahlen", die später nach ihm benannten Röntgenstrahlen.

Der 1. Internationale Tag der Radiologie nahm das Thema "Onkologische Bildgebung" in den Blick und stand unter dem Motto „Bilder helfen heilen“. Ausführliches Informationsmaterial zu diesem Thema haben die DRG und die DEGRO zu Verfügung gestellt unter:

www.drg.de

www.medizin-mit-durchblick.de

Quellen: Deutschen Röntgengellschaft Deutschen Gesellschaft für Radioonkologie 